\title{
Electrical Conduction in Polystyrene-Chloranil System
}

\author{
Y. K. KulshreshthA* and A. P. SRIVASTAVA \\ Solid State Physics Laboratory, University of Saugar, \\ Saugar (M.P.) India.
}

(Received November 26, 1979)

\begin{abstract}
The charge-transport behaviour in polystyrene-chloranil system has been studied by measuring the dependence of current on voltage, temperature, electrode material, and chloranil concentration. The experimental results are discussed in terms of the Jonscher-Ansari modified Poole-Frenkel mechanism which takes into account the effect of traps. The conductivity was found to increase.with an increase in the chloranil concentration. An explanation for this has been attempted on the basis of charge-transfer-complex formation between chloranil molecules and the phenyl group of polystyrene molecules.

KEY WORDS Poole-Frenkel Mechanism / Jonscher-Ansari Modification / Hopping Conduction / Charge-Transfer Complex /
\end{abstract}

The broadest method that can be applied to enhance the conductivity of polymeric materials is the formation of charge-transfer (CT) complexes. The electrical conduction behaviour of a number of polymer CT complexes has been investigated recently, ${ }^{1-4}$ yet the mechanism of conduction and the role of impurity in these systems is not known to any degress of certainty.

Slough $^{5}$ reported the formation of CT complex of polystyrene (PS) when an electron acceptor substance like chloranil (Chl) was introduced into its structure. The present paper describes the chargetransport mechanism in the PS-Chl system, observing the variation in current under different conditions of field, temperature, electrode material, and chloranil concentration. A model is proposed for the mechanism for enhancing conduction. It is suggested that chloranil occupies interstitial positions between the polymer chains and assists in carrier transportation by reducing the hopping barriers. Our experimental data support this model.

\section{EXPERIMENTAL}

The commercial PS supplied by $\mathrm{M} / \mathrm{s}$. Impex Chemicals, Bombay, was used in this experiment.

\footnotetext{
* Permanent address: Department of Physics,
} Dayan and Vedic College, ORAI (U.P.), India.
CT complex of PS and chromatographicallypurified $\mathrm{Chl}$ was formed by dissolving the desired amounts of the two compounds together in cyclohexanone at room temperature over a period of days. The method of film formation and geometry of vacuum-coated, sandwiched, and guarded electrodes were essentially the same as in previous papers. ${ }^{6 a, b}$ A Keithley 600B electrometer was used to record the current.

\section{RESULTS AND DISCUSSION}

Figure 1 shows the characteristics $(I-V)$ of different dopant concentration films with temperature as the parameter. These characteristics follow the relationship of the form $\log I \propto V^{1 / 2}$ and thus predict either the Richardson-Schotiky (R-S) emission-limited mechanism from the electrodes or the bulk-limited Poole-Frenkel (P-F) effect. The later effect is caused by thermal ionization of trapped electrons from donor-like centres in the presence of an external field. Table I gives the theoretical values of the $\mathrm{P}-\mathrm{S}$ coefficients $\left(\beta_{\mathrm{RS}}\right)$ and $\mathrm{P}-\mathrm{F}$ coefficients $\left(\beta_{\mathrm{PF}}\right)$ along with the experimental values of $\beta$. The dielectric constant was assumed to be 2.55 , which is a high-frequency dielectric constant for P-S. The thickness of the film was determined by capacity measurements to be about $15 \mu \mathrm{m}$ for all concentrations. Evidently, the ex- 
perimental values lie between the two theoretical values and their closeness to theoretical $\beta_{\mathrm{RS}}$ values gives the impression that process is controlled by an electrode-limited R-S mechanism. However, certain workers ${ }^{7,8}$ have noted that the wrong conclusions may be drawn regarding the applicability of the two processes on the basis of numerical values of $\beta$. The reason for this is that in some cases, $\beta_{\mathrm{PF}}$ may become even equal to $\beta_{\mathrm{RS}} \cdot{ }^{9}$ Jonscher and Ansari ${ }^{10}$ suggest

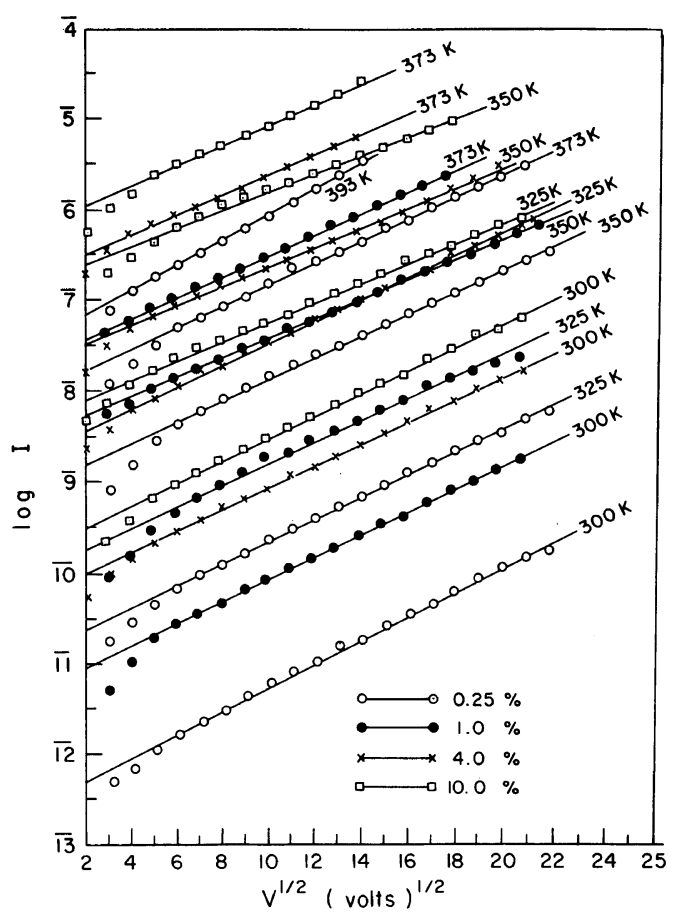

Figure 1. $\log I v s . v^{1 / 2}$ for four different concentrations of chloranil. that the basic difference between the two processes (one is electrode-limited while the other is not) may be successfully utilized for selecting the mechanism, actually involved. Consequently, graphs are plotted between $\log I$ vs. $V^{1 / 2}$ at $300 \mathrm{~K}$ for different dopant concentrations (Figure 2) using three different pairs of electrodes in each case. The electrodes with $\mathrm{Al}$, $\mathrm{Ni}$, and $\mathrm{Pb}$ were made to be negative while the other electrode $\mathrm{Al}$ was assigned a positive polarity. The plots trace a single straight line for each concentration. This behaviour indicates that the electrode-limited R-S mechanism is not the controlling mechanism in the present case.

But even the P-F model of conduction cannot be applied in its conventional form in the present investigation. The conventional model assumes a field-assisted thermal excitation of electrons from traps situated in the conduction band of insulator. Once the electron has acquired sufficient energy for reaching the conduction band, it is free to move unhindered and unobstructed. But Mott and Davis $^{11}$ point out that a drift mobility less than about $1 \mathrm{~cm}^{2} \mathrm{~V}^{-1} \mathrm{~s}^{-1}$ cannot correspond to this unhindered free-hand conduction. A modified model for conduction has been proposed by Jonscher and Ansari ${ }^{10}$ for substances of low mobility. This model inolves the use of a large number of localized trap states, randomly distributed in space and energy. Mott and Davis ${ }^{11}$ also support the existence of trap states in disordered structures. In polymers, these traps may come about as a result of regular chain folding, their (chains) termination, the presence of crystalline-amorphous interfaces, chain entanglements, etc. The new model suggests that the process of field assisted ionization of donor-like

Table I. The theoretical values of the polystyrene coefficients $\left(\beta_{\mathrm{RS}}\right)$ and $\mathrm{P}-\mathrm{F}$ coefficients $\left(\beta_{\mathrm{PF}}\right)$ along with the experimental values of $\beta^{\mathrm{a}}$

\begin{tabular}{|c|c|c|c|c|c|c|}
\hline \multirow{3}{*}{$\frac{\text { Temperature }}{\mathrm{K}}$} & \multirow{3}{*}{$\frac{\text { Theoretical } \beta_{\mathrm{RS}}}{\mathrm{V}^{-1 / 2}}$} & \multirow{3}{*}{$\frac{\text { Theoretical } \beta_{\mathrm{PF}}}{\mathrm{V}^{-1 / 2}}$} & \multirow{2}{*}{\multicolumn{4}{|c|}{$\frac{\text { Experimental } \beta}{\mathrm{V}^{-1 / 2}}$}} \\
\hline & & & & & & \\
\hline & & & $0.25 \%$ & $1 \%$ & $4 \%$ & $10 \%$ \\
\hline 300 & 0.230 & 0.460 & 0.290 & 0.275 & 0.280 & 0.285 \\
\hline 325 & 0.215 & 0.430 & 0.275 & 0.255 & 0.260 & 0.255 \\
\hline 350 & 0.200 & 0.400 & 0.265 & 0.230 & 0.230 & 0.230 \\
\hline 373 & 0.190 & 0.380 & 0.255 & 0.250 & 0.240 & 0.230 \\
\hline 393 & 0.175 & 0.350 & 0.255 & - & - & - \\
\hline
\end{tabular}

\footnotetext{
a $\mathrm{V}$, volt.
} 


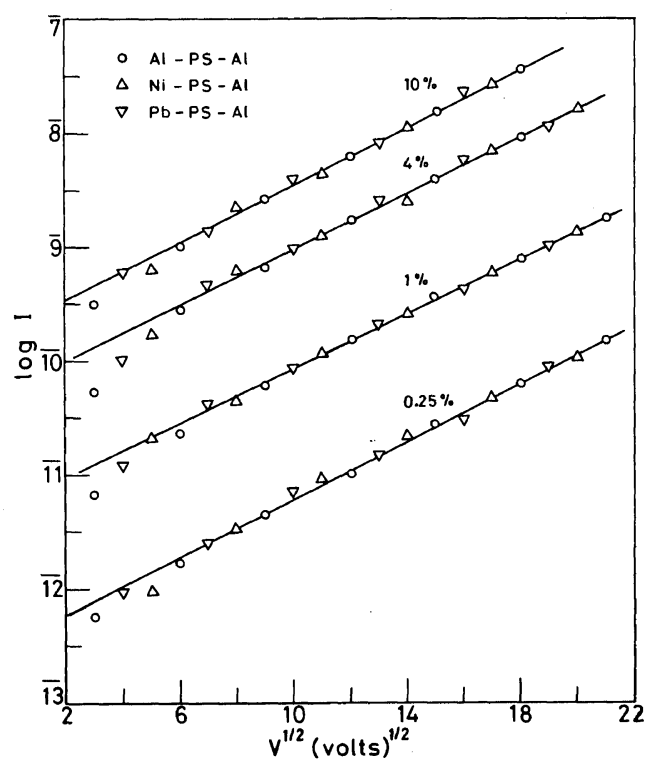

Figure 2. $\log I$ vs. $v^{1 / 2}$ for all four concentrations with three different pairs of electrodes. Some points have been left out for the sake of clarity.

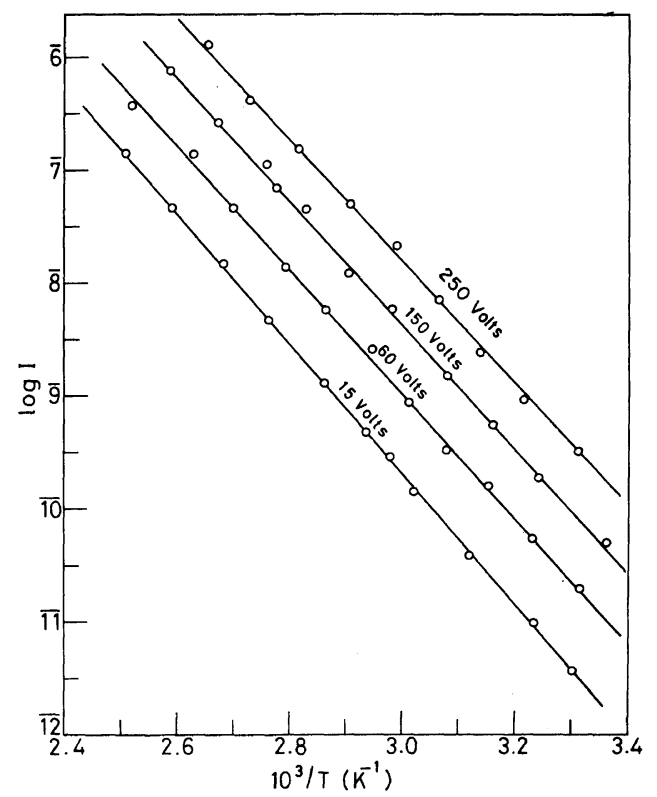

Figure 3. $\log I$ vs. $10^{3} / T$ for $0.25 \%$ doped films. centres which is the basis of $\mathrm{P}-\mathrm{F}$ mechanism, does not produce free carriers in a conduction band as is normally considered to be the case but merely causes hopping like movements of localized carriers in the trap levels. The presence of an external field assists in the process of hopping by reducing the "hop barriers" by $\gamma E^{1 / 2}$ where $\gamma=\left(e^{3} / \pi \varepsilon \varepsilon_{0}\right)^{1 / 2}$, as in the conventional $\mathrm{P}-\mathrm{F}$ model. The revised expression is written as

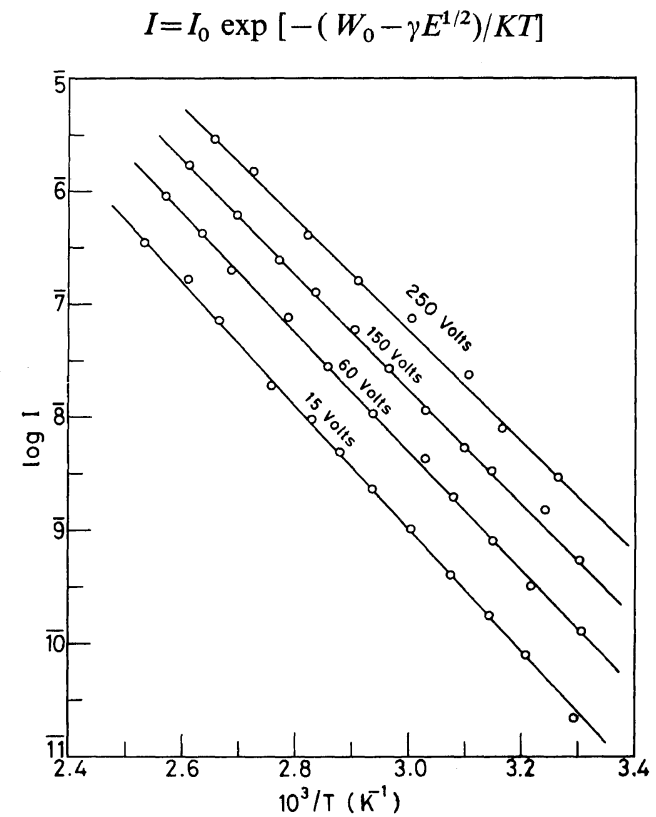

Figure 4. $\log I$ vs. $10^{3} / T$ for $1.0 \%$ doped films.

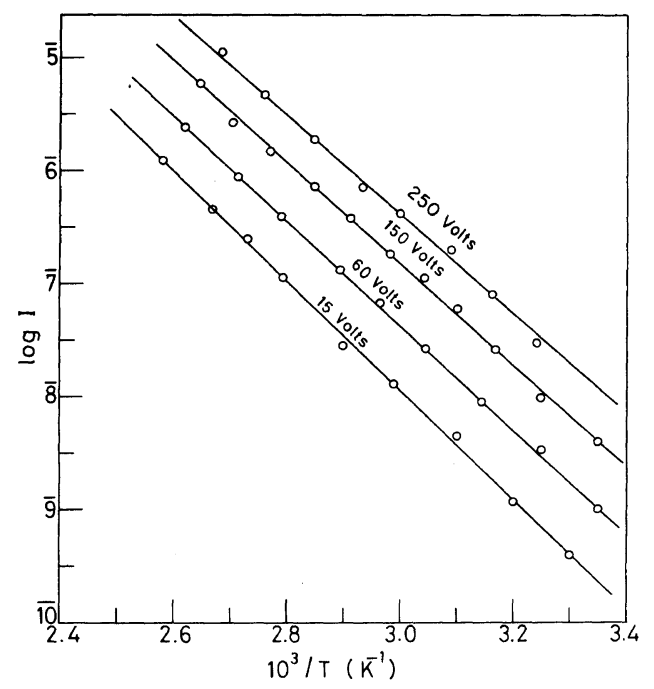

Figure 5. $\log I$ vs. $10^{3} / T$ for $4.0 \%$ doped films. 
where $W_{0}$ is the average barrier height of "hops," $I_{0}$ is the pre-exponential coefficient and may be defined as the current at an infinite temperature, $e$ is the electronic charge, $\varepsilon$ is the high-frequency dielectric constant, and $\varepsilon_{0}$ is the permittivity of free space.

To determine the values of $W_{0}$ and $\gamma$ graphs have been plotted for $\log I$ vs. $10^{3} / T$ for all the four concentrations (Figures 3-6). The activation energies calculated from the slopes of these curves have been plotted against the square root of voltage (Figure 7). A straight line was obtained for each concentration. This behaviour is consistent with the modified P-F equation. Intersepts of these lines at zero field give $W_{0}$ while the slopes determine the $\gamma$. Both of these data are listed in Table II along with

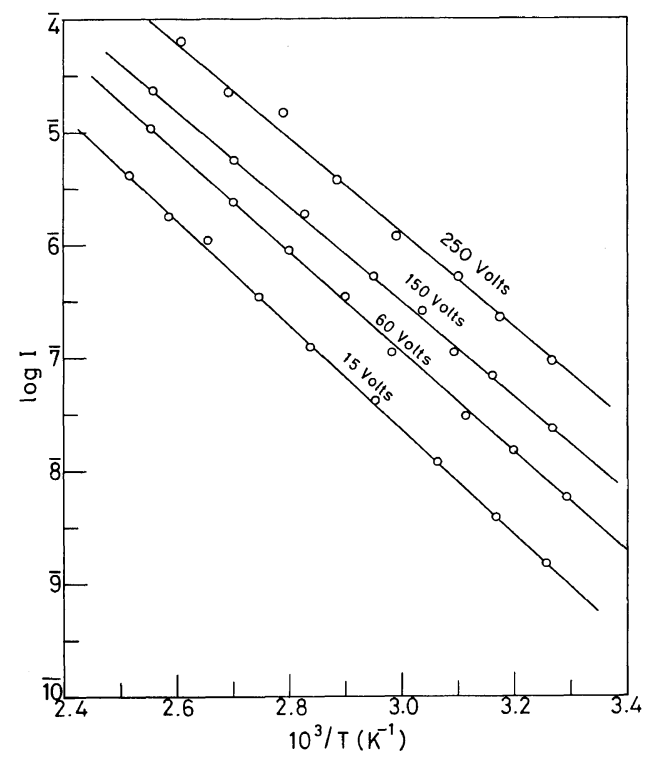

Figure 6. $\log I$ vs. $10^{3} / T$ for $10.0 \%$ doped films. the theoretical value of $\gamma$. A fair agreement between the theoretical and experimental values of $\gamma$ once again supports the dominance of the modified P-F mechanism in the present study.

Enhancement of conductivity with an increase in Chl concentration may be explained on the basis of the charge-transfer (CT) complex formation. According to the classification of Mulliken, ${ }^{12}$ PSChl CT complexes are weak complexes since both PS and Chl are aromatic systems involving $\pi-\pi$ interaction. This is also supported by the Brieglab ${ }^{13}$ criteria, according to which weak complexes are formed when $E_{\mathrm{i}}-E_{\mathrm{a}} \geqslant 5.5 \mathrm{eV}$ where $E_{\mathrm{i}}$ is the ionization potential of donor and $E_{\mathrm{a}}$ is the electron affinity of the acceptor. In the present case, the donor-acceptor action is likely to take place between the benzene ring of PS molecules and the acceptor Chl molecules. ${ }^{14}$ The value of $E_{\mathrm{i}}$ for benzene is $9.25 \mathrm{eV}$ and $E_{\mathrm{a}}$ for $\mathrm{Chl}$ is $1.37 \mathrm{eV}$. In a weak complex, there is little transfer of charge and the

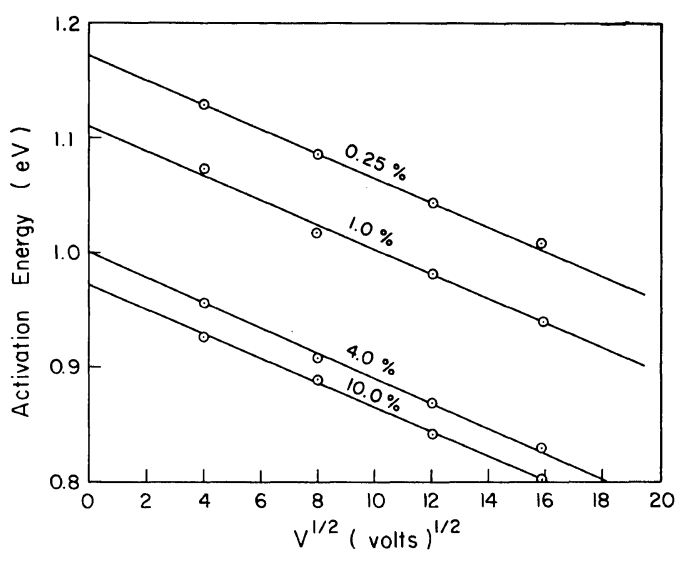

Figure 7. Activation energy vs. the square root of applied voltage for the four concentrations.

Table II. The average barrier height of "hops" $\left(W_{0}\right)$ and theoretical $\gamma$ values

\begin{tabular}{|c|c|c|c|}
\hline \multirow{2}{*}{$\begin{array}{l}\text { Concentration of } \\
\text { chloranil }\end{array}$} & Theoretical & Experimental & $W_{0}$ (From Figure 7) \\
\hline & \multirow{2}{*}{$\mathrm{JM}^{1 / 2} \mathrm{~V}^{-1 / 2}$} & \multirow{2}{*}{$\mathrm{JM}^{1 / 2} \mathrm{~V}^{-1 / 2}$} & \multirow[t]{2}{*}{$\mathrm{eV}$} \\
\hline$\%$ & & & \\
\hline 0.25 & $7.6 \times 10^{-24}$ & $6.2 \times 10^{-24}$ & 1.17 \\
\hline 1.00 & - do- & $6.6 \times 10^{-24}$ & 1.11 \\
\hline 4.00 & $-\mathrm{do}-$ & $7.0 \times 10^{-24}$ & 1.00 \\
\hline 10.00 & - do- & $6.8 \times 10^{-24}$ & 0.97 \\
\hline
\end{tabular}

a $\mathrm{V}$, volt. 
molecules are bonded by some type of weak interacting forces, much weaker than those of ionic or covalent bonding.

The conductivity of a system is enhanced by either an increase in the carrier concentrations or the mobility of the carriers or both. In the case of weak CT complex, the idea of the generation of new charge carriers on a large scale seems unreasonable. The factor contributing most to the increase in the conductivity is likely the increase in the mobility of the carriers. Davies ${ }^{15}$ has noticed an increase of about four orders of magnitude of carrier mobility when iodine was introduced into the structure of polyethylene. A similar increase in electron mobility was observed by $\mathrm{Gill}^{16}$ in poly( $N$-vinylcarbazole)trinitrofluorenone CT complex.

In the absence of any direct mobility data on Chl doped PS, the exact effect of Chl cannot be known. But still, we may conceive of this effect in the following manner. The molecular configuration of commercial PS is atactic and hence is constituted to a considerable extent by non-crystalline material. But the possibility of a few crystallites embeded in the amorphous mass cannot be precluded. X-ray analysis reveal that the impurities are contained mostly in the amorphous region of the polymer. Hence, it is reasonable to assume that $\mathrm{Chl}$ molecules occupy the interstitial positions between the polymer chains of amorphous phase and link, these chains to some kind of bonds by a charge-exchange process between the $\mathrm{Chl}$ molecule and side phenyl group of PS. This process reduces the interstitial barriers and increase the transition probability of electrons hopping across these barriers. The interfacial barriers probably also decrease (should some crystallites be present) because of the presence of $\mathrm{Chl}$ at the interfaces. Thus, the whole structure of PS will be more open for charge transportation following impregnation with $\mathrm{Chl}$. The continually decreasing average barrier height $\left(W_{0}\right)$ (Table II) and increasing conductivity (Figure 8 ) with increasing $\mathrm{Chl}$ concentration support, this reasoning.

\section{CONCLUSIONS}

1. The $I-V$ and $I-T$ data of the present study fit fairly well with the Poole-Frenkel mechanism of conduction, modified by Jonscher and Ansari.

2. The presence of Chloranil in the polystyrene structure enhances its conductivity. This has been

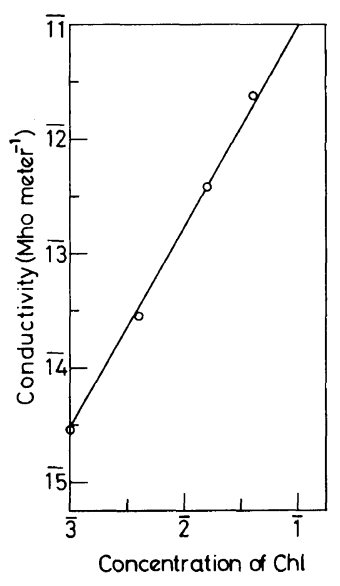

Figure 8. Conductivity $v s$. chloranil concentration in a mole-mole ratio of polystyrene to chloranil with respect to monomer units.

explained on the basis of charge-transfer-complex formation. Reduction in the average "hop" barrier supports this explanation.

\section{REFERENCES}

1. A. C. Rastogi and K. L. Chopra, Thin Solid Films, 26, 61 (1975).

2. T. J. Lewis and D. M. Taylor, J. Phys. D: Appl. Phys., 5, 1664 (1972).

3. D. W. Swan, J. Appl. Phys., 38, 5051 (1967).

4. W. Klopffer and H. Rabenhorst, J. Chem. Phys., 46, 1362 (1967)

5. W. Slough, Trans. Faraday Soc., 58, 2360 (1962).

6. (a) Y. K. Kulshrestha and A. P. Srivastava, Polym. J., 11, 515 (1979).

(b) Y. K. Kulshrestha and A. P. Srivastava, accepted for publication in Thin Solid Films.

7. M. Ieda, G. Sawa, and S. Kato, J. Appl. Phys., 42, 3737 (1971).

8. A. K. Jonscher, Thin Solid Films, 1, 213 (1967).

9. P. J. Reucroft, S. K. Ghosh, and D. Kever, J. Polym. Sci., Polym. Phys., 10, 2305 (1972).

10. A. K. Jonscher and A. A. Ansari, Phil. Mag., 23, 205 (1971).

11. N. F. Mott and E. A. Davis, "Electronic Processes in Non-Crystalline Materials," Claredon Press, Oxford, 1971.

12. R. S. Mulliken, J. Chem. Phys., Phys.-Chem. Biol., 61, 20 (1967).

13. A. Brieglab, Chemie (Int. Ed.), 3, 617 (1964).

14. M. Kryzewski, J. Polym. Sci., Polym. Lett. Ed., 4, 595 (1966)

15. D. K. Davies, J. Phys. D: Appl. Phys., 5, 162 (1972).

16. W. D. Gill, J. Appl. Phys., 43, 5033 (1972). 